\title{
Using Geographic Information Systems and the World Wide Web for Interactive Transit-Trip Itinerary Planning
}

\author{
Brian L. Smith \\ University of Virginia
}

\begin{abstract}
$\overline{\text { Abstract }}$
Providing high-quality service information is important in attracting and retaining public transportation passengers. Currently, information provided to support transit-trip itinerary planning is either difficult to use or to access. Furthermore, after obtaining the information, passengers are required to perform a fairly complex search process to extract an itinerary. Two technologies that are becoming commonly used in public transportation, Geographic Information Systems (GIS), and the World Wide Web (WWW), offer capabilities to provide an easily accessible, highly functional tool for trip itinerary planning. This research effort investigated the feasibility of integrating these emerging technologies to produce a transit-trip itinerary planning tool.
\end{abstract}

\section{Introduction}

Passenger information is an essential element of public transportation service. While the development of Intelligent Transportation Systems (ITS) has brought significant attention to new forms and types of passenger information (e.g., parking availability at transit stations and real-time system status information), "traditional" passenger information, particularly the system route and 
schedule, plays a central role. In order to attract new passengers, potential riders must have access to route and schedule information so they can identify service options that best meet their requirements. In addition to retaining riders and enabling efficient system operation, public transportation customers must have a sound understanding of the system routes and schedule. Finally, not only is the content of this information critical, the presentation also plays a key role in the perception of transit service.

The current state-of-the-practice in providing transit route/schedule information is printed brochures and interactive telephone service. While each of these approaches offers important strengths, they also suffer from significant weaknesses. In particular, printed brochures require transit customers to manually complete the significant search effort required to "extract" a trip itinerary suitable to their needs. In addition, the accessibility of telephone service is limited; it is based on time of day and, potentially, location of the transit customer.

GIS and the WWW are two information technologies that are playing an ever-increasing role in public transportation. Vendors in these areas have made significant strides to allow for the integration of these technologies. The premise of this research is that GIS and the WWW-when used together-will provide a powerful means to disseminate route and schedule information, and will serve as an ideal foundation for an automated tool to assist transit customers in planning trip itineraries.

This article explores the challenges of providing system route and schedule information. It examines how GIS and the WWW can be used to address these challenges, and describes a case study of developing such a system. Finally, the article concludes with a discussion that contrasts the process of designing a web-based GIS application to the more traditional GIS analysis process commonly used in public transportation.

\section{Traditional Approaches in Providing Route and Schedule Information}

Traditionally, route and schedule information has been presented in two formats: printed brochures and interactive telephone service.

Generally, printed brochures depict routes in stylized maps and present 
schedules in a tabular fashion. Smaller systems often include all routes and schedules in one brochure, while larger systems print multiple brochures to cover regional subsets of their system.

Interactive telephone service simply involves a customer calling a transit agency representative and providing his or her origin and destination locations, as well as time requirements: Then, the transit representative searches for a trip itinerary that best meets the customer's requirements. Some telephone services include software support to assist the transit representative in searching for an itinerary, while others simply require the representative to search standard printed route and schedule information. In all cases, the use of a telephone service supplements standard route and schedule brochures.

These traditional approaches have served transit customers well due to a number of strengths. First, printed brochures are "portable," providing customers with complete information to use in making decisions at their convenience. Also, they require little to no support from transit agencies' staff. On the other hand, the strength of the telephone service is that it provides immediate, interactive assistance to transit customers. Customers who may have difficulty deciphering the brochure can interact with a transit representative to identify a suitable trip itinerary-a process similar to working with a travel agent to purchase airline tickets.

Unfortunately, each approach also suffers from significant weaknesses. Printed brochures are static information dissemination tools. They require that the transit customer manually complete the complex analysis necessary to "extract" an itinerary that meets his or her needs. In addition, if a change is made to a route or schedule, the brochures must be modified and reproduced. Not only is this costly, but it also results in the risk that a large portion of an agency's customers will continue to rely on outdated brochures to identify trips in a system with a new route/schedule structure. Finally, it is very difficult to clearly describe special transit services (e.g., peak-period "express" service or specialized tourist routes) in a general-purpose brochure.

Telephone services also suffer from serious weaknesses. First, a customer can access the service only when a transit representative is "on duty." By 
expanding the availability of the service temporally, the transit agency is faced with higher operating costs. In addition, a customer from out of the area (e.g., a business traveler or tourist) will not likely have access to the customer service telephone number, preventing him or her from "prescheduling" a trip before traveling to the region. Finally, given that the service relies purely on verbal communications, it provides no visual information (such as a map) to the customer. If the customer is unfamiliar with the region, it may be difficult or impossible for a transit representative to "describe" verbally where a particular stop is located.

Clearly, there is room for improvement in the provision of route and schedule information. In particular, a service that retains the strengths of brochures and telephone service, while eliminating their weaknesses is most desirable. Before delving into a proposed technical approach to accomplish this, it is important to understand the core functions required for the selection of a transit-trip itinerary.

\section{Functions Required for Identifying Trip Itineraries}

While on the surface the identification of a transit-trip itinerary may seem trivial, it is, in fact, a complex process that requires a substantial amount of information and sophisticated analyses. The core functions of this process can be classified into two general categories: spatial decisions and temporal decisions.

\section{Spatial Decisions}

The first spatial decision one must make in selecting an itinerary is to determine which stop, if any, is sufficiently close to the point of origin. This decision includes basic distance measurement to determine if a stop is reasonably near the point of origin. However, beyond this, one must also take into account impediments between the origin and the stop, such as private property, bodies of water, and interstate highways. Because of these impediments, the distance measurement may change from a strictly Euclidean distance, to a shortest-path network problem.

To further complicate this decision, there is not one set "rule of thumb" that can be used to determine sufficient "closeness." Different people are willing to 
walk different distances to access transit. Furthermore, even the same person will use different distance thresholds on different days based on factors such as the weather. Finally, the assumption that the only way to access the stop is through walking is erroneous. Transit customers may bicycle to the stop or take some other form of on-demand public transportation such as a taxi service.

Once a set of potential stops has been selected that is sufficiently accessible from the user's origin, the next key spatial decision is "Can I get to where I want to go (my destination) from this origin?" The first step in making this decision is to repeat the origin search process by searching for stops that are sufficiently near the customer's destination. Again, this search must account for the complexity introduced by potential impediments between the bus stop and the destination. Once this set of potential destination stops has been identified, the next step is to determine if one can reach the stops in this set from the stops in the origin set.

In order to determine if one can reach a destination stop from an origin stop, it is necessary to search the transit system's route structure. Clearly, if the stops are on the same route, the trip is feasible. However, in many cases a series of transfers will allow one to access a destination stop on a different route from the origin stop. While this search process is not necessarily conceptually complex, it can be time consuming. For example, in an urban system, it is not beyond the realm of possibilities that 7 stops meet the minimum criteria for both the origin and destination of a trip. In this case, one would have to search 49 potential trips to examine their feasibility.

\section{Temporal Decisions}

Once the spatial decision process is complete, a transit customer is left with a number of trips that meet his or her minimum spatial requirements. The next step in selecting an itinerary is to determine which of these trips, if any, best meet his or her temporal requirements. In general, these requirements can be: (1) when the customer would like to leave the origin, (2) when the customer would like to arrive at the destination, or (3) a maximum time the customer is willing to accept for the overall trip time. In addition, in most cases, while one of these three requirements is of primary importance to a customer, the "true" 
objective usually is a combination of the three. This results in a complex, multiobjective temporal search process.

The temporal search process is, again, not conceptually complex, but it can be extremely tedious. Essentially, for each trip, one must search through the transit schedule table to identify the itinerary that best meets the customer's requirements for each origin/destination pair. In some cases, the itinerary will clearly fall short of the customer's requirements (e.g., a trip that requires 4 hours and 5 transfers). However, in general, it is likely that a number of itineraries will remain following this search process.

Finally, a number of miscellaneous factors are likely to enter the temporal search process. For example, some transit services, such as those provided for peak-period "express" service and special events, are only available during certain days or certain hours of the day. Furthermore, many transit services run modified schedules on weekends. Depending on the day and time the customer would like to travel, the temporal search must also identify which services are available to the customer.

\section{Problem Formulation}

One can consider the selection of a transit-trip itinerary in the context of a mathematical programming problem. The problem can be expressed as:

Minimize: Total trip time

Subject to: Spatial contraints

Temporal constraints

System constraints

In order to address this complex optimization problem, transit customers are generally provided with only a printed brochure. Furthermore, the problem is not deterministic. Clearly, transit-trip time is stochastic, which further complicates the problem. Thus, as demonstrated above, there is a need to provide transit customers with more effective tools to assist in selecting trip itineraries.

\section{Technology}

As shown in the previous section, the task of identifying a transit-trip itin- 
erary involves complex and time-consuming analyses. Furthermore, an ideal tool to assist transit customers in this task would be widely available at very low cost. This section provides general background on GIS and the WWWtechnical tools that provide the functionality needed to address this problem.

\section{GIS}

In his textbook, Fundamentals of Geographic Information Systems, DeMers defines GIS as a tool that supports the processing of spatial data into information (DeMers 1997). This definition clearly distinguishes GIS from the common misperception that it is simply a map graphic generator. The ability to support spatial analysis, while also simultaneously allowing for the management and analysis of large quantities of attribute data describing the spatial entities, makes GIS well suited to support the identification of transit-trip itineraries, in this case, the information in DeMers's definition.

Furthermore, GIS is currently used extensively by public transportation agencies primarily to support planning functions. For example, a recent survey of transit agencies published in the Urban Transportation Monitor reveals that agencies use GIS to support route planning, ridership market analysis, origin/destination demand analysis, and facilities management (1999). This extensive use of GIS is particularly advantageous because most transit agencies will likely possess the data and software required to serve as the foundation for a transit-trip itinerary planner.

To further explore the applicability of GIS to this problem, consider some of the fundamental spatial analyses required by a transit-trip itinerary planner. As stated earlier, proximity analysis is key to determine if a candidate bus stop serves as a viable origin or destination point. The basic GIS tools of "overlays" and "buffers" provide the needed functionality to support this requirement. For example, once a customer's point of origin is located, GIS can be used to see if that point falls within a user-defined distance from a bus stop (buffer). Furthermore, the use of a map-based GIS interface allows a customer to determine if certain objects comprise unacceptable barriers to reaching a potential transit stop.

Nearly all commercially available GISs are built on a relational database management system (RDBMS). The RDBMS serves as the engine that supports the management of attribute data describing the spatial entities. This capability 
provides the functionality needed to store and analyze schedule information. For example, for each point representing a bus stop location, attribute data are stored describing the scheduled times for buses to arrive at that stop. Therefore, once a stop is identified as potentially meeting a customer's spatial needs as described above, the RDBMS portion of the GIS supports a query to determine if the stop meets the customer's temporal requirements.

\section{WWW}

The analytical capabilities of GIS are of little use if there is no way for customers to access them. It is this wide access that the WWW provides. The web is a fundamental component of the world's modern communication network. It has many positive attributes, including:

- the ability to distribute information globally;

- accessibility to a growing market;

- interactivity;

- flexibility to revise, change, and update information at low cost; and

- the capability to include user-friendly graphics.

Global Information Distribution. The ability to distribute information globally is an important characteristic of the WWW due to the sheer size of the communication medium. Geography does not limit the feasibility of distributing information to customers on a worldwide scale. A practical example is providing transit information to tourists: travelers can plan their itinerary at a vacation destination before leaving home. With the Web, transit information is readily available to persons in another state or country.

Accessibility to a Growing Market. WWW usage is growing at an unprecedented rate. When compared to television, radio, and printed materials, the exposure of the web is significantly larger and continuing to increase. For example, traffic on the Internet-which is correlated to web usage-is growing by 20 percent every month (Forsythe, Grose, and Ratner 1998). Also, by the year 2001, approximately 174.5 million people will have access to the web (Allen, Kania, and Yaeckel 1998). Currently many economically disadvantaged individuals, a large portion of transit customers, do not have access to the web. Experts agree, however, that the phenomenal growth of the web will provide access to all population segments in the very near future. 
Interactivity. Interactivity is a key strength of the WWW. Unlike brochures, a website allows a user to question, decide, and learn through participation. This form of communication is more successful than contemporary methods because the user has direct control over the exposure to the material. In fact, it has been argued that a "website isn't something people read, it's something they do" (Sterne 1995).

Flexibility. A website possesses remarkable flexibility, allowing revisions and updates at a fraction of the time and cost of reprinting material. Unlike written materials, websites can use customer responses to quickly revise the site. Many consumers find it beneficial to complete surveys knowing that their opinions could make positive changes. Web polls are cost-effective ways to determine customer interests. Even if the website is not perfect at the outset, it can be quickly improved, provided designers are willing to have a longer-term commitment.

Graphics Capability. An additional website strength lies in its ability to include user-friendly graphics. Many people have a much easier time understanding information if it is displayed with a map or picture. If it is designed in a user-friendly manner, then the customer has control over what information is shown rather than a simple static table displaying set information. It is often easier to provide detailed, color graphics via the Web than through a printed medium.

\section{GIS and the WWW}

Until very recently, GIS and the WWW have been distinct technologies that could not be integrated. However, GIS software vendors have introduced new tools that allow GISs to become "web-enabled." In other words, these products provide access to spatial information and analysis using the WWW as the user interface. Examples of such products are ESRI's ArcView and MapObjects Internet Map Server software, and MapInfo's MapXSite software. These new products have attracted significant attention in the developer's community, and new applications are appearing almost daily.

However, the challenge to apply the new GIS/WWW capabilities lies in the performance requirements of a transit itinerary planner. Most GIS/WWW 
applications to date are relatively simple, allowing a user to request a particular static map via a WWW user interface. Relatively little analytical capabilities are included in these applications. Therefore, the question remains, "Can the current GIS/WWW tools support the level of interactivity and analysis required for a transit-trip itinerary planner?"

\section{Design Case Study}

In order to determine if GIS and the WWW could be used to serve as the foundation for a transit-trip itinerary planner, a research team designed a prototype system for the Peninsula Transportation District Commission (Pentran), the public transportation provider in the Peninsula region of southeastern Virginia. The design process allowed the team to investigate the capabilities of the available GIS/WWW tools. In addition to reporting on the case study, this section highlights a number of important differences between this application and more traditional GIS analyses conducted in public transportation.

In typical GIS applications in public transportation, an analyst works in what can best be described as a "scientific discovery" mode. Data are collected, processed, and then analyzed in an attempt to discover new information. In bus route planning, for example, socioeconomic data, ridership data, and transportation data are collected and then analyzed simultaneously to determine if areas of a region are being inadequately served or overserved. This process works very well for off-line "planning" GIS applications where an experienced analyst searches for information.

Alternatively, a GIS/WWW trip itinerary planner will be used by transit customers with no GIS experience. In addition, there are significant processing time constraints that must be met. In other words, rather than approaching the trip itinerary planner design from a "scientific discovery" perspective, the planner must be designed as a piece of applications software. Because of this, it is important to take a new, more structured approach to GIS applications design.

Software design is an area of great debate in the software engineering community. There are two general classes of software design approaches: waterfall and rapid prototyping (Eisner 1997). 
In the waterfall approach, system development follows a structured linear approach:

1. Functional requirements of the system are fully detailed.

2. A technical design is developed to meet the requirements.

3. The software is crafted.

The rapid prototyping approach essentially moves quickly through the waterfall methodology a number of times, developing more and more detailed prototype systems as the number of iterations increase. Because of this iterative nature, the rapid prototyping approach is also referred to as "spiral methodology." Both the waterfall and rapid prototyping approaches have been used successfully and unsuccessfully in the past. A complete description of the strengths and weaknesses of the approaches, while beyond the scope of this paper, can be found in the software engineering literature.

Given the uncertain nature of the technical capabilities of GIS/WWW tools, the research team chose to use the rapid prototyping approach. This allowed the team to frequently check to see if the functionality required by a particular design was provided by the tools. The core tools used in the effort were Microsoft's VisualBasic and ESRI's MapObjects Internet Map Server.

Development of the system required a significant amount of effort. The fundamental result of the effort was that the GIS/WWW tools available do provide the needed capabilities to support a transit-trip itinerary planner service.

\section{Importance of a Detailed Design}

When developing the prototypes, it became very clear that a thoroughly detailed design was essential to ensure tight integration of system components. As discussed earlier in this article, the trip planning process is complex, and this complexity resulted in a lengthy design document. Figure 1 displays the design flowchart for the web page that allows a user to input the origin location. The prototype that resulted from this design is displayed in Figure 2. Arriving at the final design and prototype displayed in these figures required six rapid prototyping iterations. 


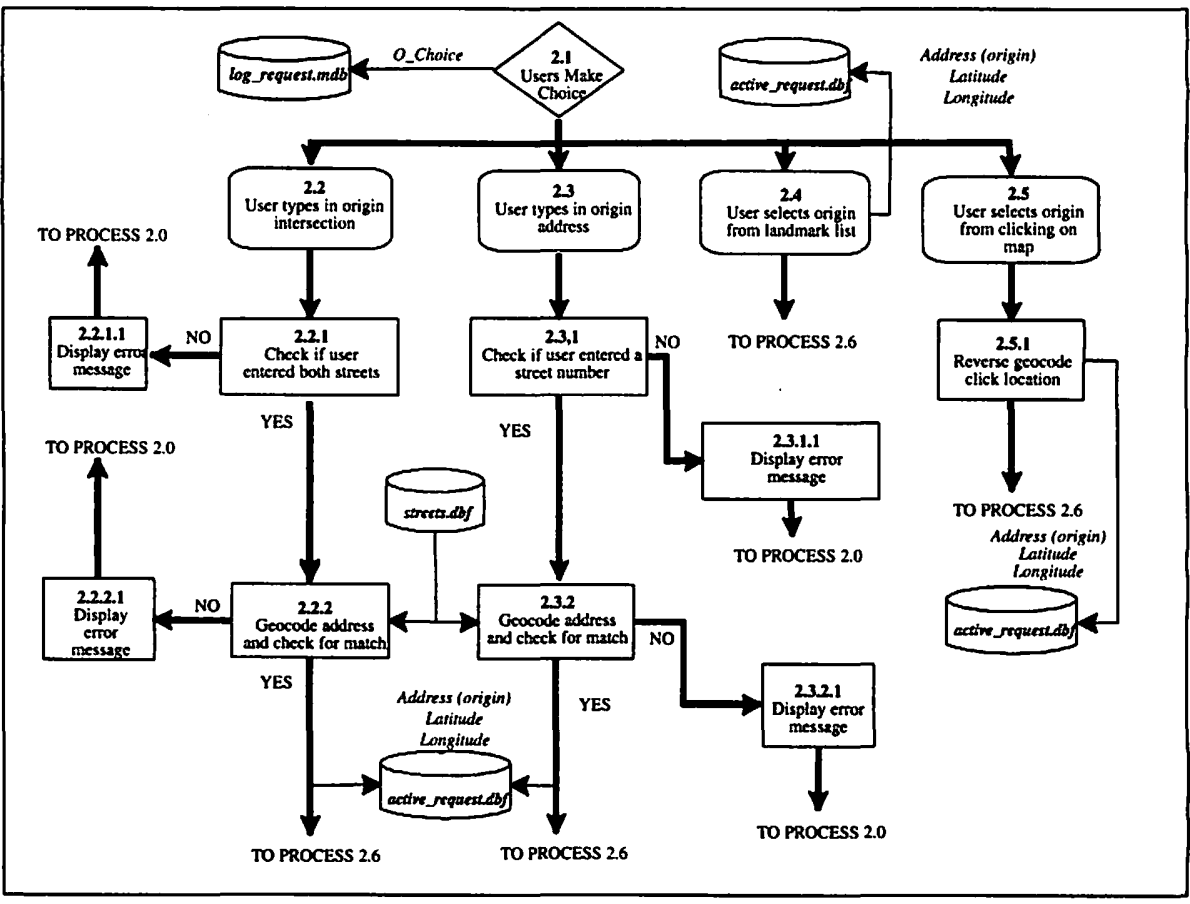

Figure 1. Design for origin input page

\section{Role of the Database Structure}

The database plays a key role in the trip itinerary planner system. It organizes data describing the transit system, and provides a mechanism to store and manage the preferences of the user as he or she interacts with the system. Considerable effort was required when designing the structure of the database tables (the schema). In fact, nearly one-third of the entire system design document is devoted to the database schema.

\section{Design for Ease of Maintenance}

As discussed earlier, one of the key advantages offered by the use of the WWW is that the trip itinerary planner can quickly adapt to changes in the transit system's routes and/or schedules. However, to allow for this, the prototype design had to directly address maintenance tasks. By considering maintenance of the system throughout the design process, changes can be made easily through databases, without changing the core source code. In addition, a portion of the design document is dedicated to detailing procedures for basic maintenance activities such as adding/deleting stops, adding/deleting routes, etc. 


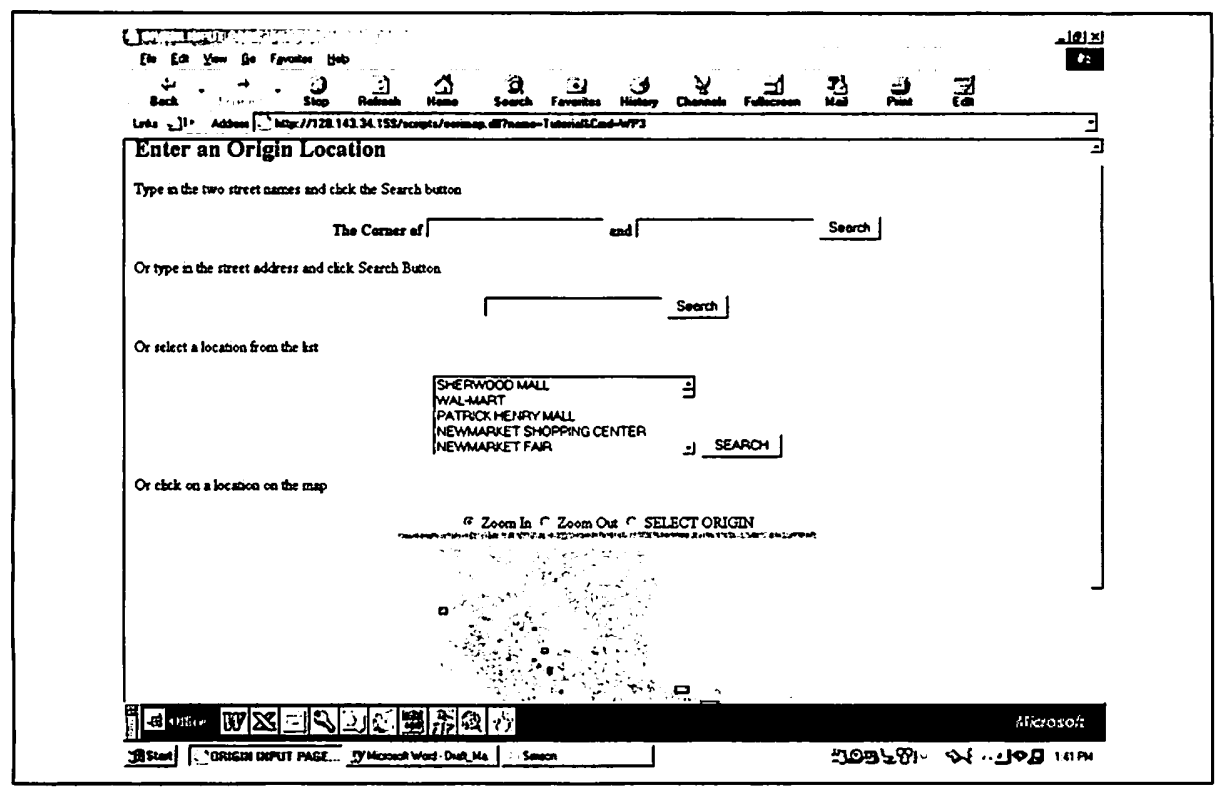

Figure 2. Prototype origin input page

\section{Conclusions}

The integration of GIS and the WWW provide capabilities needed to provide an effective tool in assisting transit customers in the complex task of selecting a trip itinerary. The development of such a tool, while feasible, requires a significantly different approach from traditional transit GIS applications. However, as shown in this research, by applying a structured software design approach, such a tool can be effectively developed.

\section{Acknowledgments}

The research reported in this article was funded by Pentran and the MidAtlantic Universities Transportation Center. The results described here are due in large part to the efforts of other members of the Pentran research team at the University of Virginia: Brian Revels, Kevin Smith, and John Miller.

\section{References}

Allen, C., D. Kania, and B. Yaeckel. 1998. Internet world guide to one-to-one Web marketing. New York: John Wiley and Sons, Inc.

DeMers, M. N. 1997. Fundamentals of Geographic Information Systems. New York: John Wiley and Sons, Inc. 
Eisner, H., 1997. Essentials of project and systems engineering management. New York: John Wiley and Sons, Inc.

Forsythe, C., E. Grose, and J. Ratner. 1998. Human factors and Web development. London: Lawrence Erlbaum Associates.

Sterne, J. 1995. World Wide Web marketing: Integrating the Internet into your marketing strategy. New York: John Wiley and Sons, Inc.

Urban Transportation Monitor. March 19, 1999. 13(5): 8-11.

\section{About the Author}

BRIAN SMITH (briansmith@virginia.edu) is an assistant professor in the Department of Civil Engineering at the University of Virginia. Dr. Smith's research interests are Intelligent Transportation Systems, transportation systems management, and advanced information technology applications. 